\title{
A Néoplasma of the Breast Revealed by a Bilateral Choriodal Metastasis
}

\author{
Souhail Hassane ${ }^{1,2, \text { * }}$, Ifrkhas Said ${ }^{1,2}$, Laktaoui Abdelkader ${ }^{1,2}$ \\ ${ }^{1}$ Department of ophthalmology, Military hospital, Meknes Morocco \\ ${ }^{2}$ Faculty of medicine and pharmacy, Fes Morocco
}

Email address:

souhai126@yahoo.fr (S. Hassane)

\section{To cite this article:}

Souhail Hassane, Ifrkhas Said, Laktaoui Abdelkader. A Néoplasma of the Breast Revealed by a Bilateral Choriodal Metasytasis. Journal of Cancer Treatment and Research. Vol. 3, No. 3, 2015, pp. 28-31. doi: 10.11648/j.jctr.20150303.11

\begin{abstract}
The uveals metastases are the most frequent malignant tumours of the eye, 10 times more than the melanoma. Their rate of which has occurred is estimated in the recent literature between 2 and 38\%, their period of apparition are more often at the final stage of the neoplasma. Our work present the rare case of 40 year old patient, in quite condition general, addressed to our consultation for bilateral retinal detachment, whose clinical exams, angiographic, B-scan, CT-tomography and mammography make it possible to keep the diagnosis of bilateral uveal metastasis revealing an unrecognised néoplasma of the breast to the stage of bony and ganglial metastasis. Knowing the severe prognosis of uveal metastasis, a suitable treatment is started trying as well as possible to preserve the visual prognosis in this patient suffering of cancer.
\end{abstract}

Keywords: Choroïd, Metastasis, Breast Cancer

\section{Introduction}

Described the first time in 1864 by Horner, choroidals metastasis constitutes the most frequent malignant tumours of the eye of adult. There frequency it esteemed to be $2 \%$ to $7 \%$ $(1,2)$ these choroidals metastasis appear with a variable time after the discovery of primary sites of 24 to 60 month. These choroidals metastasis appear generally at a final stage of neoplasma and coexist with some metastasis of an other organs in 60 with $90 \%$ of case. However, they can be revealing of cancer (in $20 \%$ of case), the diagnosis of uveal metastasis precede the discovery of primary tumour in $55,5 \%$ of bronchial carcinoma and in $4,9 \%$ in mammary carcinoma (3) by L.ZOGRAFOS on 98 cases of uveal metastasis in 1992, as well as the series of STEPHENE and CHIELDS (4), and sometimes the origin remains ignored in $8 \%$ of the cases. The characteristic of our observation rests on the revealing character of the breast cancer of these bilateral choroidal metastasis.

\section{Observation}

A 40 years old women was referred to us for bilateral retinal detachment, the functional signs are represented by decreased visual acuity in $8 / 20$ in the left and right eye with alteration in field of vision. In slit-lamp examination, the anterior segment is normal. The ocular motility is normal, Fundus examination showed (fig. 1, 2), a inferotemporal dome-shaped creamy yellow choroidal tumor associated with a serous retinal detachment in both eyes. B scan revealing an echogenic choroidal with a high internal reflectivity mass with a retinal detachment in tow eyes (fig 3). Fluorescein angiography revealed late hyperfluorescence with pinpoint foci of hyperfluorescence over the tumor (fig. 4). With indocyanine green, the choroidal hyperfluorescence is more visible (fig. 5). Given the character little prominent of the process, the bilateralism, the appearance of ophtalmoscopy, the angiographic aspect of the lesion; we evoke a secondary localisation, that imposes a cheek-up in search of the primitive neoplasma and a cheek-up of extension. So a pulmonary $\mathrm{x}$-ray, abdominal ultrasound scan, a bony scintigraphy, a mammography, revealed the existence of a breast cancer with the bony and ganglial metastasis. The patient is addressed and treated by gynecologists and oncologists she was operated of her neoplasma of breast and completed by sittings of chemotherapy and local radiotherapy. The anatomo-pathological exam recovers an canalr adenocarcinoma. 


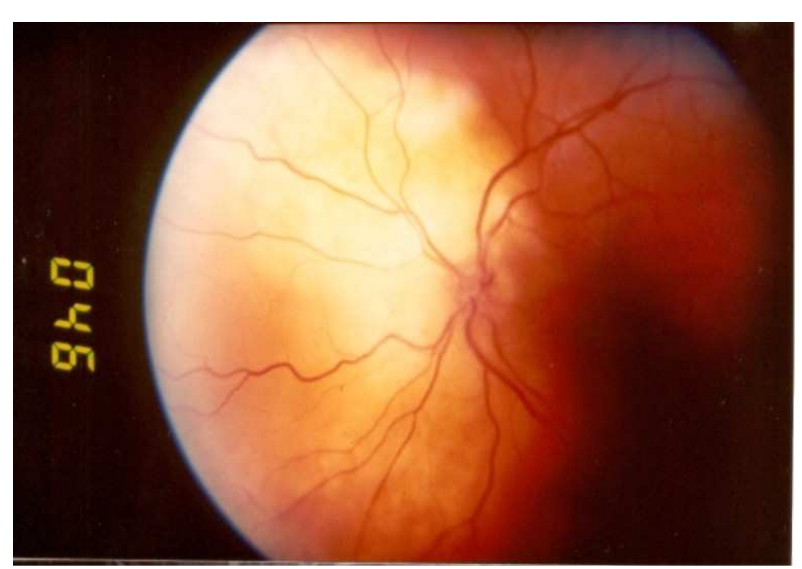

Fig. 1.

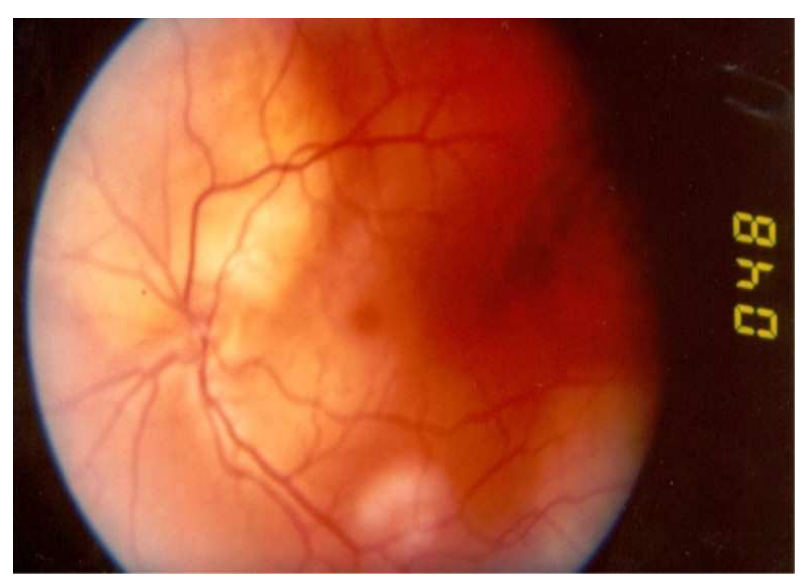

Fig. 2.

Fig. 1, 2. fundoscopy showing Choroidal metastasis.

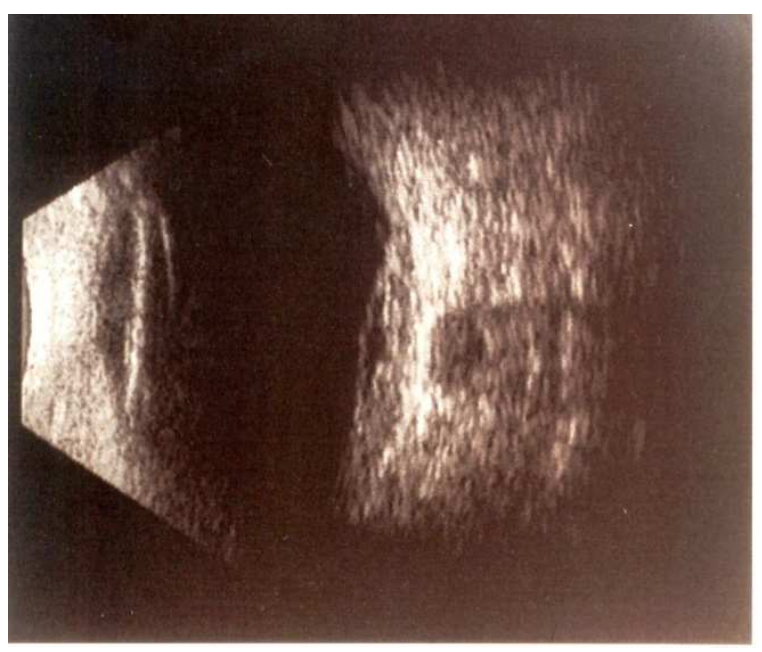

Fig. 3. B scan ultrasonography showed a choroidal mass pattern with moderate internal reflectivity with an exudative $R D$.

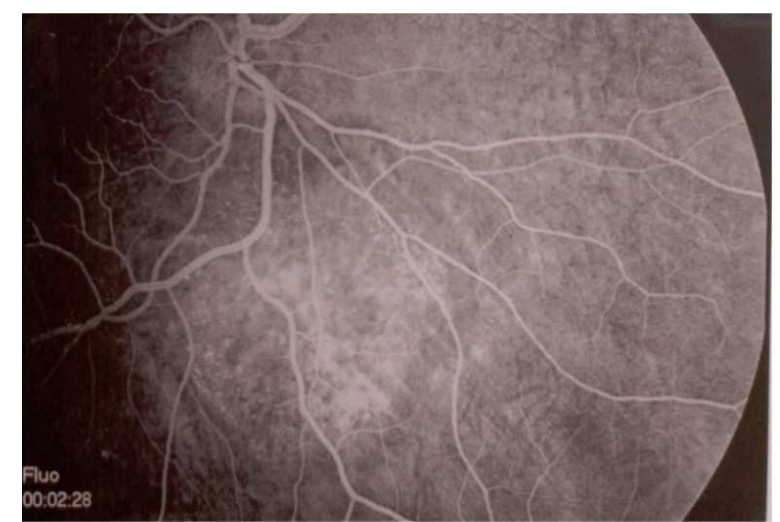

Fig. 4. A choroidal tumor showed late hyperfluorescence in the inferior temporal quadrant, with pinpoint foci of hyperfluorescence over the tumor.

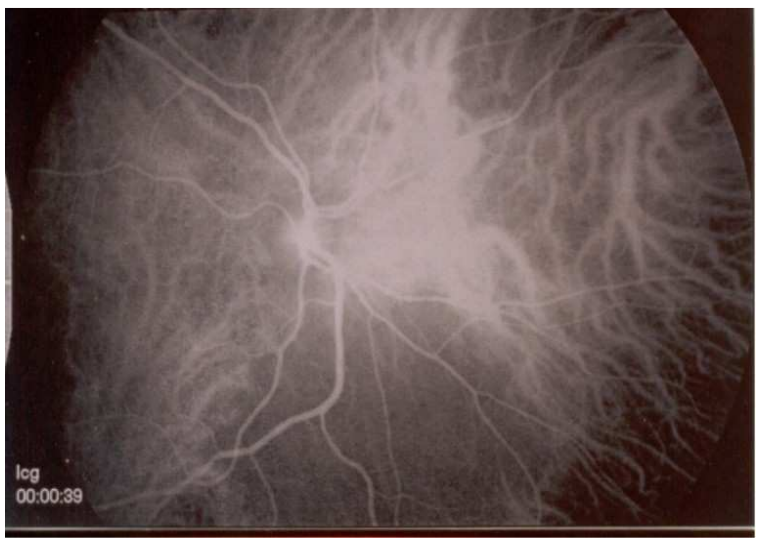

Fig. 5. choroidal hyperfluorescence after indocyanine green injection.

\section{Comment}

While any portion of the eye can be involved by metastatic disease, the most common tissue involved is the highly vascular choroids $(1,5)$. There is no good explanation as to why the eye, particularly the choroid, is a common site for metastasis. Ferry and Font (6) speculated that the distribution of tumors within the choroid may be related to its vascularity characteristics. Also, as previously noted, there are significant differences between primary tumors and their incidence of ocular metastasis. The reasons for these differences are also unexplained, but Ferry and Font suggest that the high incidence of breast cancer metastasis may be related to the longer life expectancy of breast cancer patients with metastasis, thus providing a longer time for intraocular metastasis to develop.

Shields et al (8) surveyed 420 consecutive patients with uveal metastases. The tumors were unilateral in 320 patients and bilateral in 100 patients. This proportion of bilateral cases is considerably more than the $4.4 \%$ noted by Ferry and Font. The study by Mewis and Young (5) of breast cancer patients noted a $31 \%$ incidence of bilaterality. In both the Ferry study and the Mewis study, the incidence of subsequent bilaterality was notable $(17.6 \%$ and $15 \%$, respectively). There seems to be no predilection for metastasis to preferentially affect the right or left eye (7). 
Mewis and Young (5) analysed 250 patients with known breast carcinoma. At the time of evaluation, 152 patients were referred secondary to ocular symptoms, and 98 were asymptomatic. In the asymptomatic group, nine patients $(9.2 \%)$ were found to have metastatic disease.

although they appear at the end of a variable time after the discovery of the primary sites, the choroidal metastasis permits to be revealing of the primary cancer in $20 \%$ of cases .Most metastatic neoplasm's to the eye are carcinomas, with the breast and lung being the most common primary sites of origin. Studies by Castro, Ferry, and Shields suggest that between $16 \%$ and $18 \%$ of these lesions are of unknown primary origin $(8,9)$.

Between $52 \%$ and $70 \%$ of patients with metastatic ocular disease are women $(9,10,11)$. This finding is attributed to the high prevalence of breast cancer among women presenting with metastases to the eye (12) Upon presentation, as many as two thirds of patients with intraocular disease will have a history of cancer (9). Those with lung cancer are more likely to present without knowledge of their primary neoplasm (8).

With choroidal metastases, the most common symptom is painless loss of vision. Other presenting symptoms have been described, including photopsias, floaters, pain, redness, field defect, metamorphopsia, and diplopia. Up to $10 \%$ of patients may be asymptomatic (9). Mewis and Young diagnosed nine choroidal tumors in 98 asymptomatic women with breast cancer (13).

Although patients present with vision ranging from 20/20 to no light perception, Shields found that $45 \%$ of the patients in his series had 20/200 or worse vision (9).

Choroidal metastases are classically homogenous, creamy yellow to yellow-white subretinal lesions. They take on a flat or dome-shaped configuration with discrete borders and are often associated with a secondary serous retinal detachment (14).

Although once thought to localize preferentially in the left eye, metastatic tumors have been found to develop equally in both eyes. Bilateral disease occurs in $4 \%$ to $40 \%$ of patients, with a higher incidence observed in the setting of breast carcinoma $(15,8,10,16)$.

The hearths can be single or multiple, localised with the posterior pole in $70 \%$ and raising the retinal plan slightly being able to involve a serous retinal detachment (14).

Fluorescein angiography of metastatic lesions has a characteristic appearance, although false-positive angiograms have been described. Relative hypofluorescence is seen in the arterial and early venous phase. A progressive, mottled hyperflourescence occurs subsequently in the later venous phase, with hypofluorescent areas corresponding to clumps of brown pigment on the tumor surface. Intense hyperfluorescent, pinpoint lesions are also seen. With indocyanine green, the tumor may show diffuse late, homogeneous hypofluorescence with no leakage. Fluorescein patterns are similar for most metastatic lesions and are not useful in identifying the primary lesion. With lesions of the anterior segment, angiography provides no diagnostic assistance $(12,17)$.
The differential diagnosis of a choroidal metastasis includes choroidal amelanotic nevus, amelanotic uveal melanoma, choroidal hemangioma, choroidal osteoma, posterior scleritis, cytomegalovirus (CMV) retinitis, choroidal granuloma, choroidal neovascularization with disciform scar, and retinal detachment $(18,19)$ Perhaps the most difficult differential diagnosis involves distinguishing a primary amelanotic melanoma from a metastatic lesion.

In addition to a thorough physical examination, a systemic work-up should include a mammogram and chest radiograph, given the prevalence of breast and lung cancer. In patients with no history of cancer, up to $50 \%$ with choroidal lesions suspected to be metastatic will not have a primary tumor site identified by their oncologist (9).

Once a diagnosis is established, appropriate treatment should be initiated to preserve vision and improve quality of life. Depending on the health of the patient and status of his or her malignancy, this may take various forms.

Radiation is perhaps the most commonly employed therapy for these patients. Indications for radiation treatment of choroidal metastasis include a decrease in visual acuity or a rapidly enlarging lesion that threatens acuity (20). Often delivered by external beam, 3000 to 4000 Gy are applied in divided doses over a 2 to 4 week period. $(21,22)$ Some authors have advocated a lower dose of 2000 Gy, whereas doses as high as 5000 Gy are also found in the literature (23, 24).

Freidman and Folk reported that the median survival time of patients with breast carcinoma metastatic to the choroid was 314 days, whereas that of patients with orbital metastases was 794 days (10). Similarly, Mewis and Young found that the median survival of breast cancer patients with choroidal lesions was 9 months (20).

\section{Conclusion}

Ocular metastases from breast carcinoma remain most common The overall frequency of ocular metastasis in patients dying of cancer is high as $37 \%$. Intraocular spread usually occurs in the posterior choroid as a creamy yellow infiltrate. Patients present with painless loss of vision. The diagnosis generally can be made by careful ophthalmologic examination. Radiotherapy is the cornerstone of management and will allow the majority of patients to maintain useful vision

\section{References}

[1] De Potter P, Disneur D, Levecq L, Snyers B. Manifestations oculaires des cancers. J Fr Ophtalmol 2002;25:194-202

[2] Jyotirmay B, Thomas C Ho, Kaushal B. Bilateral metastasis to the retina, choroids and optic nerve from breast cancer: A clinicopathological case 2007; 55: 71-72

[3] Zografos L., Chamero J., Bercher L., Uffer S. Les métastases de l'uvée et leur traitement. Ophtalmologie, 1992, 6, 13-19. 
[4] Stephan RF., Shield JA. diagnostic and management of cancer metastatic to the uvea: a stady of 70 cases. Ophtalmology 1979; 86: 1336-49.

[5] Mewis L, Young SE. Breast carcinoma metastatic to the choroid: analysis of 67 patients. Ophthalmology. 1982;89:147151

[6] Ferry AP, Font RL. Carcinoma metastatic to the eye and orbit. I: A clinicopathologic study of 227 cases. Arch Ophthalmol. 1974;92:276-286.

[7] Eliassi-Rad B, Albert DM, Green WR. Frequency of ocular metastases in patients dying of cancer in eye bank populations. Br J Ophthalmol. 1996;80:125-128.

[8] G. Pugneta, S. Aristaa, G. Martin-Blondela, Choroidal metastasis as a presenting feature of breast carcinoma. Revue de médecine interne 2007; 28: 790-792.

[9] Shields JA, Shields CL, Gross NE: Survey of 520 eyes with uveal metastases. Ophthalmology , 1997; 104:1265-1276.

[10] Freedman MI, Folk JC: Metastatic tumors to the eye and orbit: Patient survival and clinical characteristics. Arch Ophthalmol, 1987; 105:1215-1219.

[11] Hutchinson DS, Smith TR: Ocular and orbital metastatic carcinoma. Annals of Ophthalmology, 1979; 11:869-873.

[12] Shields JA: Metastatic tumors to the uvea. Int Ophthalmol Clin , 1993; 33:155-161./57/

[13] Shields JA, Shields CL (eds): Intraocular Tumors: A Text and Atlas. Philadelphia, WB Saunders, 1992, pp 208-238, 489-512

[14] William Small, Jr, MD. Management of Ocular Metastasis., cancer controle, 1998; 4, 5: 350- 356.
[15] Bloch RS, Gartner S: The incidence of ocular metastatic carcinoma. Arch Ophthalmol , 1971; 85:673-675.

[16] Hart WM: Metastatic carcinoma to the eye and orbit. Int Ophthalmol Clin 1962; 2:465-482.

[17] Shields JA, Shields CL, Kiratli H, et al: Metastatic tumors to the iris in 40 patients. Am J Ophthalmol 119:422-430, 1995

[18] Michelson JB, Stephens RF, Shields JA: Clinical conditions mistaken for metastatic cancer of the choroid. Annals of Ophthalmology1979; 11:149-153.

[19] Volpe NJ, Albert DM: Metastases to the uvea. In Albert DM, Jakobiec FA (eds):

[20] Principles and Practice of Ophthalmology, ed 4, vol 5. Philadelphia, WB Saunders, 1994, pp 3260-3270

[21] Mewis L, Young SE: Breast carcinoma metastatic to the choroid: Analysis of 67 patients. Ophthamology, 1982 ; 89:147-151.

[22] Orenstein M, Anderson D, Stein J: Choroidal metastases. Cancer , 1972; 29:1101-1107

[23] Shields JA, Shields CL (eds): Intraocular Tumors: A Text and Atlas. Philadelphia, WB Saunders, 1992, pp 208-238, 489-512

[24] Dobrowsky W: Treatment of choroidal metastases. Br J Radiol, 1988; 61:140-142

[25] Reddy S, Saxena VS, Hendrickson F, et al: Malignant metastatic disease of the eye: Management of an uncommon complication. Cancer, 1981; 47:810-812. 